\title{
ANÁLISE DO PERFIL DE ADMISSÃO, EVOLUÇÃO E DESFECHO INTRA-HOSPITALAR DE IDOSOS: COORTE PROSPECTIVA
}

\author{
Thamara Graziela Flores ${ }^{1}$ \\ Melissa Agostini Lampert² \\ Gabriele Ruiz Keller \\ Diogo Oliveira de Paula \\ Erica de Freitas Alvarenga ${ }^{5}$ \\ Tassiane Moreira dos Santos ${ }^{6}$ \\ Renata Rojas Guerra?
}

\footnotetext{
1 Graduada em Fisioterapia. Mestre em Gerontologia. Doutoranda em Farmacologia na Universidade Federal de Santa Maria (UFSM). E-mail: thamaraflores_fisio@yahoo.com.br.

2 Graduada em Medicina. Doutora em Medicina e Ciências da Saúde. Professora vinculada ao departamento de clínica médica na Universidade Federal de Santa Maria (UFSM). E-mail: melissa.a.lampert@ gmail.com.

3 Graduada em Fisioterapia. Mestre em Gerontologia. Doutoranda em Ciências da Saúde na Universidade Federal do Rio Grande (FURG). E-mail: gabriele.r.k@hotmail.com.

4 Graduado em Medicina pela Universidade Federal de Santa Maria (UFSM). Email: diogooliveira21@ hotmail.com.

5 Graduada em Medicina pela Universidade Federal de Santa Maria (UFSM). Email: erica.fa@hotmail.com.

6 Graduada em Medicina pela Universidade Federal de Santa Maria (UFSM). Email: tassiianemoreira@ gmail.com.

7 Graduada em Ciências Econômicas. Doutora em Estatística. Professora vinculada ao departamento de estatística da Universidade Federal de Santa Maria. Email: renata.rojasg@gmail.com.
} 
resumo

O envelhecimento populacional é permeado por uma série de desafios para a saúde pública brasileira, desta forma a avaliação do perfil de idosos em internação hospitalar, considerando presença de comorbidades, parâmetros funcionais e presença de fragilidade tornam-se imprescindível. Dessa forma, busca-se avaliar o perfil de idosos na admissão, evolução hospitalar e desfecho e verificar a associação do perfil da admissão com o desfecho da internação. Trata-se de um estudo de coorte prospectiva. Utilizou-se como variáveis da admissão hospitalar o perfil sociodemográfico e perfil clínico-funcional, como variáveis da evolução, o tempo de internação hospitalar e complicações e como desfecho - alta hospitalar ou óbito. Realizou-se análise descritiva e teste do qui-quadrado ou teste exato de Fisher para associação das variáveis. Foram avaliados 493 idosos, com maioria de idosos jovens, que moravam sozinhos, admitidos no hospital devido a neoplasia. Houve associação positiva do óbito com a idade, Delirium, índice de Comorbidades de Charlson, risco sênior, Fragilidade e valores da circunferência de panturrilha. Verificou-se que a população idosa que ingressa em emergência hospitalar apresenta multimorbidades, declínios funcionais, fragilidade e maior exposição a eventos adversos, entre eles o óbito. Recomenda-se a elaboração de estratégias, para que o cuidado prestado nesse ambiente contemple as peculiaridades desse grupo etário, possibilitando melhores resultados de saúde.

palavras-chave

Envelhecimento. Hospitalização. Mortalidade.

As mudanças demográficas e o aumento da expectativa de vida populacional são um reflexo de avanços nas áreas da saúde e das tecnologias. No ano de 1940, tínhamos $42 \%$ da população caracterizada como jovens, com idade inferior a 15 anos, enquanto que o número de idosos era de 2,5\% da população, esse número se modificou exponencialmente para 10,8\%. Estima-se que em 2060 o número de idosos quadruplique, o que ressalta as modificações que serão exigidas, a partir desta realidade (SBGG, 2014). O aumento da expectativa de vida ocasionou uma maior predisposição às doenças crônicas não 
transmissíveis (DCNT). Essas são responsáveis por maiores gastos dos sistemas de saúde, devido principalmente à comorbidades associadas, uso contínuo de fármacos, declínios funcionais que ocasionam maior dependência e risco de hospitalização (PEREIRA, D.; NOGUEIRA; SILVA, 2015).

Sabe-se que as DCNTs foram responsáveis por 72\% dos óbitos em 2007, sendo muito destes associados à redução de reservas funcionais e consequente vulnerabilidade clínica desencadeada por internações hospitalares (DANIELEWICZ; BARBOSA; DEL DUCA, 2014). Dessa forma, vê-se como importante a busca por estratégias que visem integralidade de saúde. Sabe-se que a integralidade está relacionada à prevenção de DCNT e agravos relacionados ao envelhecimento, além de manutenção da qualidade de vida em todos os ambientes de cuidado que se façam necessários, incluindo o hospitalar (THIEME et al., 2014).

Observa-se que com o envelhecimento surge uma maior utilização de serviços hospitalares, com maior número de intervenções, custo com tratamentos, tempo de hospitalização, complexidade de atendimento e demanda por serviços de urgência e emergência, evidenciando a necessidade de uma adaptação desses serviços às novas demandas demográficas (CHODOS et al., 2015; PEREIRA, E. et al., 2014). Dados mostram que em 2017, 25,55\% dos pacientes internados tinham mais de 60 anos de idade, caracterizando cerca de 2.933.305 internações no Brasil (BRASIL, 2017).

O ambiente hospitalar está associado à um maior risco de eventos adversos, principalmente quando o período de internação é duradouro ou frequente. Entre os eventos adversos se destacam o Delirium, iatrogenia medicamentosa, infecções, imobilidade, quedas, desnutrição, úlceras por pressão e tromboembolismo venoso, todos com possíveis declínios funcionais e cognitivos associados. Em estudo de Carvalho et al. (2018), observou-se que houve declínio funcional em cerca de $28 \%$ dos idosos 30 dias após a alta, quando comparado a 15 dias antes da internação, sendo que os idosos que apresentavam indiciadores de fragilidade possuíram maior risco de devolver perda funcional (SOURDET et al., 2015; CARVALHO et al., 2018). Existem estimativas de que ocorra um declínio global de 25\% a 35\% em idosos hospitalizados (SALES; SANTOS, I., 2007).

Como um dos marcadores de qualidade de vida do idoso é a observação de sua capacidade funcional e o ambiente hospitalar é um potencial desencadeador de perdas funcionais irreversíveis, vê-se como imprescindível a utilização de parâmetros funcionais como rotina no cuidado de idosos em internação hospitalar. Destaca-se também que a avaliação da capacidade funcional pode servir como preditora do risco do idoso de apresentar eventos adversos durante a internação (SALES; SANTOS, I., 2007; CARVALHO et al., 2018; ZISBERG et al., 2015). 
Entre as variáveis que interferem na capacidade funcional dos idosos está a presença de comorbidades, sendo esta um preditor de pior desfecho intra-hospitalar, possuindo maior risco durante o decorrer da internação. Ao analisar a diferença entre os gêneros, as mulheres com maior número de comorbidades possuem maior perda funcional decorrente da internação, quando comparadas aos homens (ALMAGRO et al., 2020).

A perda de massa muscular é uma importante ferramenta na clinica médica e multiprofissional para identificar idoso propensos à patologias como a Sarcopenia e a Fragilidade, bastante prevalente no meio intra-hospitalar. Uma das formas de analisar esta váriavel é através da circunferência de panturrilha $(\mathrm{CP})$, sendo que em idosos hospitalizados os valores de $\mathrm{CP}$ menores que $31 \mathrm{~cm}$ são preditores de mortalidade, incapacidade, perda funcional e maior número de complicações (LANDI et al., 2014; PÉREZ-ZEPEDA; GUTIÉRREZ-ROBLEDO, 2016; MAEDA et al., 2017). Outro fator que repercute diretamente na evolução clínica dos pacientes idosos em ambiente hospitalar é a presença da fragilidade, que é uma patologia clínica multidimensional, definida por um estado de vulnerabilidade na resposta a fatores estressores, expondo os indivíduos a riscos como institucionalização, quedas, fraturas, maior permanência hospitalar e óbito (MORLEY et al., 2013; RITT et al., 2017).

Idosos hospitalizados apresentam maior predisposição para sintomas depressivos, alguns estudos trazem que mais de um terço dos pacientes hospitalizados exibem sintomas depressivos (CIRO et al., 2012; HAMMOND et al., 2008). Recentemente, a depressão é apresentada cientificamente como preditor de declínio funcional, quedas, readmissões e mortalidade de idosos hospitalizados (PIERLUISSI et al., 2012; GASSMANN; RUPPRECHT; FREIBERGER, 2009; CULLUM et al., 2008; REICHARDT et al., 2019).

$\mathrm{O}$ ambiente intra-hospitalar apresenta mudanças na rotina dos idosos, $\mathrm{e}$ está envolvido em eventos que diminuem homeostase dos indivíduos. Uma das consequências destas alterações está no estado de humor, como observado na depressão (REICHARDT et al., 2019), e no estado cognitivo, como a presença de Delirium. O Delirium é caracterizado como um distúrbio agudo e flutuante de atenção, consciência e cognição, com maior prevalência em idosos hospitalizados ou após cirurgias, sendo reconsiderado um preditor de morbidade, mortalidade e maior custo na internação (BAI et al., 2019; JIN et al., 2020). Um dos instrumentos utilizados na clinica médica para detectar risco de desenvolver desfecho adverso durante a internação é o Identification of Seniors at Risk (ISAR). Em estudo, Gronewold et al. (2017) identificaram que escores elevados neste instrumento em idosos internados no Departamento de Ortopedia e Cirurgia do Trauma do Hospital Universitário de Essen eram 
preditores de maior cuidados e gastos de assistência de saúde, como apoio de enfermagem e fisioterapia, bem como maior tempo de internação e comorbidades associadas a internação.

Assim, com o envelhecimento populacional envolve uma série de desafios para a saúde pública brasileira, entende-se como importante a avaliação do perfil de idosos em internação hospitalar, considerando presença de comorbidades, parâmetros funcionais e presença de fragilidade, como forma de subsidiar a elaboração de estratégias para que o cuidado prestado nesse ambiente contemple as peculiaridades desse grupo etário, possibilitando melhores resultados de saúde.

\section{Objetivo}

O objetivo deste estudo é avaliar o perfil de idosos na admissão, evolução hospitalar e desfecho, como também verificar a associação do perfil da admissão com o desfecho da internação.

\section{Métodos}

Trata-se de um recorte do projeto "Desenvolvimento de uma linha de cuidado ao idoso hospitalizado do HUSM", aprovado no comitê de ética e pesquisa com serem humanos sob parecer de número 48212915.50000.5346, referente ao período entre setembro de 2015 e outubro de 2016, no Hospital Universitário de Santa Maria/RS (HUSM). Este projeto é um estudo quantitativo, longitudinal e descritivo, constituindo uma coorte prospectiva que analisou o perfil de admissão hospitalar de idosos, o desfecho durante a internação até a alta e até 30 dias após a alta. O HUSM recebe pacientes provenientes da região central do Rio Grande do Sul, prestando uma assistência à saúde de qualidade à população do município e da região central do Rio Grande do Sul, abrangendo 42 municípios, com uma população de cerca de 1.000.000 habitantes.

A amostra para o presente estudo foi calculada através do software Epi info versão 7 para realização de estudos de coorte. Através da revisão de literatura, foi encontrada a proporção entre os indivíduos em emergências, classificados em risco ou não, através dos preditores, assim como a incidência das comorbidades em cada categoria. Considerou-se uma exposição de $25 \%$ a $50 \%$, utilizando um nível de confiança de $95 \%$, um poder estatístico de $80 \%$ e uma estimativa de perdas em torno de $20 \%$, o que gerou o cálculo de uma amostra de 546 indivíduos. Foi realizado um processo de seleção amostral 
aleatório simples e os idosos selecionados foram avaliados por acadêmicos do curso de Medicina da Universidade Federal de Santa Maria, após treinamento interno realizado pela pesquisadora responsável. Para a realização desse estudo foram seguidos os princípios éticos da Resolução CNS 466/12 que regulamenta a pesquisa com seres humanos no Brasil, onde os idosos, após ingressar na emergência hospitalar, foram convidados a participar da pesquisa, sendo esclarecido sobre riscos e benefícios de sua participação, e após o consentimento foi assinado o Termo de Consentimento Livre e Esclarecido pelo paciente ou pelo responsável.

Foram incluídos pacientes com idade igual ou superior a 60 anos, de ambos os sexos, que internaram no Pronto Socorro do Hospital Universitário de Santa Maria no município de Santa Maria/RS, independente de causa específica para internação, quando realizada a avaliação da admissão hospitalar, dentro das 48 horas de internação. Optou-se por incluir as avaliações incompletas com mínimo de 2/3 das informações necessárias, por ser considerado aceitável a dificuldade na obtenção de alguns dados, devido características próprias de um ambiente de emergência (possibilidade de instabilidade clínica, necessidade de realização de exames ou transferência que impossibilitem a realização da avaliação completa nas 48 horas da admissão). Foram excluídos os pacientes que possuíam incapacidade de responder aos questionamentos dessa avaliação (por déficit cognitivo ou de comunicação) e não tinham a presença de um acompanhante que pudesse fornecer os dados necessários; os pacientes cujas avaliações ficaram incompletas por impossibilidade de finalização dentro das 48 horas de internação (foram consideradas incompletas as avaliações com menos de 2/3 das informações necessárias).

O instrumento utilizado para a coleta dos dados na admissão hospitalar foi um formulário elaborado para esse fim, composto por dados sociodemográficos e por uma avaliação clínico-funcional. Posteriormente, para a coleta de dados da evolução hospitalar e do desfecho, foi utilizado um formulário específico para registro da ocorrência de complicações (pneumonia, quedas, infecção do trato urinário, trombose venosa profunda, Delirium e incontinência urinária), tempo de internação e ocorrência de alta hospitalar ou óbito, preenchido a partir da revisão dos prontuários, realizada pelo mesmo acadêmico, a cada 48 horas, durante todo o período de internação. Para a avaliação clínico-funcional, foram selecionados instrumentos que avaliassem a presença de comorbidades, a capacidade funcional (de forma que contemplassem aspectos de cognição, humor e mobilidade do idoso) e fragilidade.

O índice de comorbidades de Charlson (ICC) é considerado uma importante ferramenta para medir as comorbidades presentes no idoso (ALMAGRO 
et al., 2020). Foi desenvolvido em 1984 com base em pacientes hospitalares e verifica a presença de 17 comorbidades divididas em 19 situações clínicas. Cada uma das condições possui diferentes pontuações, entre 1 a 3, que predizem o risco relativo de sobrevida em um ano, sendo que quanto mais elevado o escore, maior severidade. O escore de 1 ponto representa $98 \%$ de chance de sobrevida em um ano, de 2, 3 e 4 pontos $89 \%$ de sobrevida, de 5 a 6 pontos representam $79 \%$ de sobrevida e 7 ou mais pontos a $64 \%$ de sobrevida em um ano (MARTINS, 2010).

A perda da massa muscular foi avaliada pela medida pela circunferência de panturrilha $(\mathrm{CP})$, sendo um preditor de sarcopenia (associada à presença de fragilidade), classificando os idosos em duas categorias: maior ou igual a $31 \mathrm{~cm}$ e menor que $31 \mathrm{~cm}$, como preconizado pela OMS (Organização Mundial de Saúde), sendo que valores menores que $31 \mathrm{~cm}$ predizem sarcopenia (LOHMAN; ROCHE; MARTORELL, 1988). A fragilidade foi avaliada através da escala de fragilidade de Edmonton (EFE), que é um instrumento que avalia, a partir de nove domínios e onze itens, o grau de fragilidade de pacientes submetidos ao teste. A pontuação máxima dessa escala é 17 e representa o nível mais elevado de fragilidade. Os escores para análise da fragilidade são: 0-4 não apresenta fragilidade; 5-6 aparentemente vulnerável; 7-8 fragilidade leve; 9-10 fragilidade moderada; 11 ou mais fragilidade grave (ROLFSON et al., 2006). Os indicadores de depressão foram avaliados através da escala de Depressão Geriátrica versão 4 (EDG4). A pontuação desta escala varia de 0 a 4 pontos, sendo que se o escore obtido for $\geq 1$ ponto, o indivíduo apresenta indicadores de depressão (CASTELO et al., 2009).

A Confusion Assessment Method (CAM) é considerada o instrumento diagnóstico mais eficaz para auxiliar o diagnóstico de Delirium, sendo composto por quatro atributos: 1) Início agudo e curso flutuante; 2) Desatenção; 3) Pensamento Desorganizado; 4) Alteração do Nível de Consciência. O diagnóstico de Delirium é firmado quando se identifica a presença dos itens "1" e "2" e um dos itens de "3" ou "4" (TANAKA et al., 2015). O Identification of Seniors At Risk (ISAR) é um índice de predição de risco criado com o objetivo de detectar o aparecimento de comorbidades e desfechos adversos em idosos internados em emergências (GRONEWOLD et al., 2017).

$\mathrm{O}$ instrumento consiste em seis perguntas que avaliam a capacidade funcional do idoso antes e depois do aparecimento da comorbidade, o número de hospitalizações nos seis meses anteriores à internação, a memória, a autopercepção de saúde e o uso de polifarmácia, referido como o uso de mais de três medicamentos diariamente. Idosos que possuem escores $>2$ pontos possuem alto risco de desenvolver desfechos adversos na hospitalização (TAVARES; 
GRÁCIO; NUNES, 2017). Dessa forma, foram consideradas como variáveis da admissão hospitalar o perfil sociodemográfico (idade, sexo e com quem reside) e o perfil clínico-funcional (patologia principal que gerou a internação, índice de comorbidades de Charlson, circunferência de panturriha, Escala de fragilidade de Edmonton, Escala de depressão geriátrica versão 4, Confusion Assessment Method e Identification of Seniors At Risk); como variáveis da evolução o tempo de internação hospitalar e complicações (pneumonia, imobilidade, quedas, infecções, trombose venosa profunda, Delirium e incontinência urinária); e como desfecho a alta hospitalar ou óbito.

A coleta de dados da avaliação da admissão hospitalar ocorreu na beira do leito de internação no Pronto Socorro do HUSM. Já a avaliação da evolução e desfecho ocorreu neste mesmo local ou nas demais unidades de internação, na ocorrência de transferência do paciente, a evolução das complicações e desfechos ocorreram através da evolução médica ou de enfermagem, descritas nos prontuários.

Após a coleta de dados, estes foram inicialmente plotados em tabelas do software Excel. A análise descritiva dos dados ocorreu com apresentação de média, mediana e desvio padrão de variáveis contínuas, após a verificação da curva de normalidade dos mesmos. Quanto às variáveis categóricas binárias, nominais ou de contagem discreta foi calculada a frequência. Também foi realizada a descrição dos dados ausentes (correspondentes à inclusão de avaliações incompletas com mínimo de $2 / 3$ das informações necessárias). A associação das variáveis da admissão hospitalar com o desfecho foi realizada pela análise univariada, através do teste do qui-quadrado e teste exato de Fisher. Todos esses testes estatísticos foram realizados pelo programa Statistical Package for the Social Sciences (SPSS) (versão 21.0) com intervalo de confiança de 95\% e níveis de significância menores que $5 \%$ sendo considerados estatisticamente significativos $(p \leq 0,05)$. 
Foram avaliados 533 idosos, ocorrendo à inclusão de 493 idosos, mediante a observação dos critérios de inclusão. Observou-se predominância do sexo masculino (56\%) e a idade variou entre 60 a 105 anos (média de 72 $\pm 8,78$ anos), com destaque para os idosos jovens (45\% entre 60 e 69 anos). Dos idosos que constituíram a amostra, $87 \%(\mathrm{n}=431)$ moravam com alguém. Quanto à patologia principal que gerou a internação, houve destaque para neoplasia $20 \%$ ( $n=97)$. Segundo o ICC, 39\% ( $n=191)$ dos idosos possuíam $89 \%$ de chance de sobrevida, $62,1 \%(n=306)$ apresentaram $C P>31 \mathrm{~cm}, 33,3 \%(n=164)$ não eram frágeis. Também segundo a EDG4, 53,6 \% (n=221) apresentaram indicadores de depressão, 64\% ( $\mathrm{n}=31)$ não apresentaram Delirium identificados pela CAM e, segundo o ISAR, 51,5\% ( $\mathrm{n}=23)$ tinham alto risco de desfecho adverso durante a hospitalização, demais resultados podem ser observados na Tabela 1. Através deste perfil é possível identificar que idosos jovens ingressam na emergência hospitalar com maior prevalência, quando comparados aos idosos longevos, e apresentando risco elevado de desenvolver desfechos adversos no período de hospitalização, observa-se que existe ainda o predomínio por DCNTs como causa de admissão hospitalar.

Quanto às variáveis de evolução clínica, observou-se que 57\% (n = 251) dos idosos tiveram complicações durante a internação e o tempo de internação variou entre um e 115 dias de internação (média de 14 14,96 dias). Constatou-se que 39\% $(n=190)$ permaneceram hospitalizados até uma semana. Quanto ao desfecho, 20,7\% ( $n=102)$ dos idosos tiveram óbito intra-hospitalar, conforme descrito na Tabela 2. Quanto à análise da associação das variáveis da admissão hospitalar com o desfecho, observou-se associação positiva da ocorrência de óbito com idade, CAM, ICC, ISAR, EFE e CP, conforme descrito na Tabela 2. Ao analisar os resultados destas variáveis é possível determinar que os idosos que tiveram o óbito como desfecho durante a internação, apresentavam episódio de Delirium, perda de massa muscular, maior grau de fragilização e risco de desfecho adverso. 
Tabela 1 - Perfil de admissão hospitalar, evolução e desfecho

\begin{tabular}{|c|c|c|c|c|c|}
\hline \multicolumn{3}{|l|}{ Variáveis } & \multirow{2}{*}{$\begin{array}{l}\text { Descrição } \\
\text { Feminino }\end{array}$} & \multirow{2}{*}{$\frac{n}{219}$} & \multirow{2}{*}{$\frac{(\%)}{44}$} \\
\hline ADMISSÃO & PERFIL & Sexo & & & \\
\hline & & & Masculino & 274 & 56 \\
\hline & & \multirow[t]{5}{*}{ Idade } & 60-69 anos & 224 & 45 \\
\hline & & & 70-79 anos & 168 & 34 \\
\hline & & & 80-89 anos & 79 & 16 \\
\hline & & & 90-99 anos & 20 & 4 \\
\hline & & & $>100$ anos & 2 & 1 \\
\hline & & \multirow{3}{*}{$\begin{array}{l}\text { Mora com } \\
\text { quem }\end{array}$} & Alguém & 431 & 87 \\
\hline & & & Sozinho & 49 & 10 \\
\hline & & & |LPI & 11 & 2 \\
\hline & \multirow{13}{*}{$\begin{array}{l}\text { PERFIL } \\
\text { CLÍNICO- } \\
\text {-FUNCIONAL }\end{array}$} & \multirow{7}{*}{$\begin{array}{l}\text { Motivo de } \\
\text { internação }\end{array}$} & Neoplasias & 97 & 19,7 \\
\hline & & & Fraturas & 95 & 19 \\
\hline & & & Digestivo & 88 & 18 \\
\hline & & & AVC & 87 & 18 \\
\hline & & & Outros & 51 & 11 \\
\hline & & & Infecto & 39 & 8 \\
\hline & & & Pulmonar & 36 & 7 \\
\hline & & \multirow[t]{4}{*}{$\begin{array}{l}\text { Impacto de } \\
\text { comorbidades }\end{array}$} & $\begin{array}{l}98 \% \text { chance de } \\
\text { sobrevida }\end{array}$ & 0 & 0 \\
\hline & & & $\begin{array}{l}89 \% \text { chance de } \\
\text { sobrevida }\end{array}$ & 191 & 39 \\
\hline & & & $\begin{array}{l}79 \% \text { chance de } \\
\text { sobrevida }\end{array}$ & 185 & 37 \\
\hline & & & $\begin{array}{l}\text { 64\% chance de } \\
\text { sobrevida }\end{array}$ & 117 & 24 \\
\hline & & \multirow{2}{*}{$\begin{array}{l}\text { Circunferência } \\
\text { de panturrilha }\end{array}$} & $\geq 31 \mathrm{~cm}$ & 130 & 26,4 \\
\hline & & & $<31 \mathrm{~cm}$ & 306 & 62,1 \\
\hline
\end{tabular}




\begin{tabular}{|c|c|c|c|c|c|}
\hline \multicolumn{3}{|l|}{ Variáveis } & \multirow{2}{*}{$\begin{array}{l}\text { Descrição } \\
\text { Não frágil }\end{array}$} & \multirow{2}{*}{$\frac{n}{164}$} & \multirow{2}{*}{$\begin{array}{l}(\%) \\
33,3\end{array}$} \\
\hline ADMISSÃO & PERFIL & Fragilidade & & & \\
\hline & $\begin{array}{l}\text { CLINICO- } \\
\text {-FUNCIONAL }\end{array}$ & & Levemente frágil & 148 & 30 \\
\hline & & & $\begin{array}{l}\text { Moderadamente } \\
\text { frágil }\end{array}$ & 153 & 31 \\
\hline & & & Frágil & 28 & 5,7 \\
\hline & & Indicativo de & Sim & 221 & 53,6 \\
\hline & & & Não & 191 & 46,4 \\
\hline & & Presença de & Não & 318 & 64 \\
\hline & & & $\operatorname{Sim}$ & 92 & 19 \\
\hline & & $\begin{array}{l}\text { Risco de } \\
\text { desfecho } \\
\text { adverso em } \\
\text { emergência }\end{array}$ & $\begin{array}{l}\text { Baixo risco } \\
\text { Alto risco }\end{array}$ & $\begin{array}{l}224 \\
239\end{array}$ & $\begin{array}{l}48,4 \\
51,6\end{array}$ \\
\hline \multirow{5}{*}{\multicolumn{2}{|c|}{ EVOLUÇÃO CLÍNICA }} & Complicações & Não & 189 & 43,0 \\
\hline & & & $\operatorname{Sim}$ & 251 & 57,0 \\
\hline & & Tempo de & 1 semana & 190 & 39 \\
\hline & & & 2 semanas & 159 & 32 \\
\hline & & & $>2$ semanas & 144 & 29 \\
\hline \multirow{4}{*}{\multicolumn{2}{|c|}{ DESFECHO }} & Alta & $\operatorname{Sim}$ & 391 & 79,3 \\
\hline & & & Não & 102 & 20,7 \\
\hline & & Óbito & Sim & 102 & 20,7 \\
\hline & & & Não & 391 & 79,3 \\
\hline
\end{tabular}

Fonte: Elaborada com base no banco de dados dos autores (2016).

Nota: ILPI: Instituição de Longa Permanência para Idosos. 
Tabela 2 - Análise da associação entre variáveis da admissão hospitalar com o desfecho

\begin{tabular}{|c|c|c|c|c|}
\hline & Variáveis & Descrição & \% óbito (n) & $p$ \\
\hline \multirow[t]{10}{*}{ PERFIL DEMOGRÁFICO } & \multirow[t]{2}{*}{ Sexo } & Feminino & $8.92(44)$ & \multirow[t]{2}{*}{0.7694} \\
\hline & & Masculino & $11.76(58)$ & \\
\hline & \multirow[t]{5}{*}{ Idade } & 60-69 anos & $6.49(32)$ & \multirow[t]{5}{*}{0.0004} \\
\hline & & 70-79 anos & $7.10(35)$ & \\
\hline & & 80-89 anos & $5.68(28)$ & \\
\hline & & 90-99 anos & $1.42(7)$ & \\
\hline & & $>100$ anos & $0.00(0)$ & \\
\hline & \multirow[t]{3}{*}{ Mora com quem } & Alguém & 18.05 (89) & \multirow[t]{3}{*}{0.0984} \\
\hline & & Sozinho & $1.62(8)$ & \\
\hline & & |LPI & $1.01(5)$ & \\
\hline \multirow{13}{*}{$\begin{array}{l}\text { PERFIL } \\
\text { CLÍNICO-FUNCIONAL }\end{array}$} & \multirow{7}{*}{$\begin{array}{l}\text { Motivo da } \\
\text { internação }\end{array}$} & Neoplasias & $5.48(27)$ & \multirow[t]{7}{*}{0.4092} \\
\hline & & Fraturas & 2.64 (13) & \\
\hline & & Digestivo & 3.65 (18) & \\
\hline & & AVC & $3.45(17)$ & \\
\hline & & Outros & $2.23(11)$ & \\
\hline & & Infecto & $1.83(9)$ & \\
\hline & & Pulmonar & $1.42(7)$ & \\
\hline & \multirow[t]{4}{*}{ ICC } & $\begin{array}{l}98 \% \text { chance de } \\
\text { sobrevida }\end{array}$ & $1,6(8)$ & \multirow[t]{4}{*}{0.003} \\
\hline & & $\begin{array}{l}89 \% \text { chance de } \\
\text { sobrevida }\end{array}$ & $39,2(40)$ & \\
\hline & & $\begin{array}{l}79 \% \text { chance de } \\
\text { sobrevida }\end{array}$ & $7,3(36)$ & \\
\hline & & $\begin{array}{l}64 \% \text { chance de } \\
\text { sobrevida }\end{array}$ & $3,7(18)$ & \\
\hline & \multirow[t]{2}{*}{$\mathrm{CP}$} & $\leq 31 \mathrm{~cm}$ & $11,2(49)$ & \multirow[t]{2}{*}{0.0016} \\
\hline & & $>31 \mathrm{~cm}$ & 26,4 (130) & \\
\hline
\end{tabular}




\begin{tabular}{|c|c|c|c|c|}
\hline & Variáveis & Descrição & $\%$ óbito (n) & $p$ \\
\hline \multirow{10}{*}{$\begin{array}{l}\text { PERFIL } \\
\text { CLIINICO-FUNCIONAL }\end{array}$} & EFE & Não Frágil & $5,7(28)$ & 0,001 \\
\hline & & $\begin{array}{l}\text { Levemente } \\
\text { Frágil }\end{array}$ & $3,9(19)$ & \\
\hline & & $\begin{array}{l}\text { Moderada- } \\
\text { mente Frágil }\end{array}$ & $8,9(44)$ & \\
\hline & & Frágil & $2,2(11)$ & \\
\hline & EDG4 & Sim & 9,5 (39) & 0.5721 \\
\hline & & Não & 90,5 (454) & \\
\hline & CAM & Sim & $2.43(74)$ & 0.0024 \\
\hline & & Não & 191 & \\
\hline & ISAR & Alto risco & 48,5 (239) & 0.0001 \\
\hline & & Baixo risco & $45,4(224)$ & \\
\hline
\end{tabular}

Fonte: Elaborada com base no banco de dados dos autores (2016).

Nota: $p<0,00$.

\section{Discussão}

Atualmente, está ocorrendo um acelerado envelhecimento populacional em países de desenvolvimento, assim como no Brasil. É previsto que, em 2040, cerca de 23,8\% da população brasileira tenha 60 anos ou mais (MENDES et al., 2012; SANTOS, C.; BESSA; XAVIER, 2020). Essas mudanças na pirâmide etária ocorrem devido ao balanço entre natalidade e mortalidade, e cuja responsabilidade pode ser traçada a partir do controle de doenças infecto parasitárias na infância e dos avanços nas tecnologias assistidas de saúde médica e hospitalar e pelo perfil de morbidade populacional. Estes parâmetros, principalmente de morbidade, estão relacionados à concepção do envelhecimento e a forma com que as DCNTs estão influenciando na qualidade de vida dos idosos, em ênfase no adoecimento, incapacidades, morbidades e mortalidade dessa população.

Este estudo tem como característica ser pioneiro na descrição do perfil de idosos que ingressaram na emergência hospitalar ao analisar variáveis sociodemográficas, clínico-funcionais e desfechos durante a internação até a alta hospitalar. Foi identificado que a faixa etária de idosos que tiveram a 
prevalência de ingresso foi de 60 aos 69 anos, com média de 72 \pm 8,78 anos, semelhante ao encontrado no estudo de Reis et al. (2008), que também encontrou predomínio de idosos jovens (até 70 anos), podendo este dado ser referente ao predomínio das patologias de internação que ocorreram neste estudo, onde as causas de maior prevalência foram por neoplasias, fratura de fêmur (19\%) e por problemas do trato digestório, patologias mais prevalentes em idosos jovens.

Quanto ao sexo, houve predomínio de indivíduos do sexo masculino (56\%), dado corroborado em diversos estudos (PINHEIRO et al., 2002; PAPALÉO NETTO et al., 2005; COSTA-JÚNIOR; MAIA, 2009; SANTOS, L. et al., 2010; CHODOS et al., 2015). Segundo Papaléo Netto et al. (2005), esse panorama se justifica devido ao comportamento preventivo da mulher, que possibilita o diagnóstico precoce de patologias no nível primário de atenção à saúde, diferente do que ocorre com os homens, que buscam atendimento mais tardiamente, necessitando de outros níveis de atenção à saúde, com maior necessidade de internações hospitalares.

Da amostra analisada, 87\% ( $n=431)$ moravam com algum familiar ou cônjuge, semelhante aos dados encontrados por Cesar et al. (2008), onde 78,2\% dos idosos residentes na cidade de Caracol no Piauí moravam com alguém. Este dado leva ao encontro do apoio familiar ao cuidado aos idosos da amostra. Entretanto, uma porcentagem significativa de idosos morava sozinha (10\%), o qual nos remete aos desafios que estão por vir, com famílias menores, idosos independentes, mas com multimorbidades associadas a velhice e os papéis da família, da sociedade e do governo frente a estas mudanças epidemiológicas.

O principal motivo de internação foi por neoplasias (20\%), seguida de fratura de fêmur (19\%), por problemas do trato digestório (18\%), e por acidente vascular cerebral (AVC) (18\%). Diferentemente do que foi analisado em pesquisa realizada por Motta et al. (2001), onde a primeira causa de hospitalização de idosos foi por doenças do aparelho digestivo (23\%), seguida de doenças do aparelho circulatório (18\%) e neoplasias (11\%). Em investigação de Pinheiro et al. (2002), realizada no Rio de Janeiro, o principal motivo de internação constatado foi de doença cardiovascular (33\%). Já em Belém, com o estudo de L. Santos et al. (2010), a principal causa de internação foi por doenças respiratórias (54\%).

Com relação às diferenças na prevalência das patologias observadas, vê-se como importante para a análise desse tipo de estudo a consideração de características regionais. Nesse contexto, destaca-se o papel do HUSM como referência na região central do Rio Grande do Sul no atendimento nas áreas de oncologia e traumatologia.

Sabe-se que a internação é um importante recurso para tratamento de idosos que necessitem de atenção especializada, mas também é de conhecimento 
que internações prolongadas ou repetidas podem levar a consequências negativas na saúde dos idosos, como aumento da fragilidade, diminuição da qualidade de vida e perda da capacidade funcional (SILVA et al., 2012). No presente estudo, o tempo médio de internação foi de 14 dias, sendo que 29\% permaneceram hospitalizados por mais de duas semanas. Comparando a estudos semelhantes realizados por Silva et al. (2012) e L. Santos et al. (2010), vê-se dados semelhantes, com uma média de internação de 17 dias e 16 dias, respectivamente.

Quanto ao impacto das comorbidades, de acordo com o ICC, observou-se que $12,6 \%(n=62)$ apresentavam $64 \%$ de chance de sobreviver em ano, observou-se também que 100\% ( $\mathrm{n}=493)$ dos idosos apresentaram mais de uma comorbidade, corroborando com estudo de Sousa-Muñoz et al. (2013), em que 92,3\% da amostra de idosos residentes do Rio de janeiro, em situação pós-hospitalização, também apresentavam mais de uma comorbidade. Segundo Miyata et al. (2008), em estudo com idosos japoneses, observou-se maior chance de óbito em idosos hospitalizados que apresentavam comorbidades associadas ao quadro clínico, verificado através do ICC, sendo que os escores que mais tiveram associação também foram os de igual ou superior a dois pontos, como o que foi observado neste estudo.

A utilização da CP para identificar perda de massa muscular é reforçada por estudos como de Pérez-Zepeda e Gutiérrez-Robledo (2016), que, além de identificar presença de perda muscular nos indivíduos avaliados, observou a associação da $\mathrm{CP}$ com a predição de incapacidade, necessidade de cuidados e mortalidade, o que também foi identificado no estudo de Landi et al. (2014) e no presente estudo.

A fragilidade também é considerada uma síndrome consequente do envelhecimento e do desequilíbrio homeostático do organismo. No estudo de Ensrud et al. (2007), com cerca de 9.704 idosas com fratura de fêmur, foram classificadas quanto a fragilidade pela presença de três ou mais dos seguintes critérios: perda de peso não intencional, fraqueza, energia pobre auto-relatada, baixa velocidade de marcha e baixa atividade física e, dessa forma, identificou-se que as idosas que eram frágeis ou pré-frágeis, independente de idade, tinham maior risco de morte, corroborando com os dados encontrados neste estudo.

Identificamos que $66,7 \%(n=329)$ dos idosos apresentavam algum grau de fragilidade, como já mencionado por Freire et al. (2017). Idosos com fragilidade possuem como desfechos principais durante a internação maior tempo de internação, reinternação, transferências e perdas psicossociais, físicas e/ou funcionais. $\mathrm{O}$ que nos traz a reflexão sobre a forma com que a fragilidade 
pode ser prevenida, visto que possui impactos importantes no decorrer do envelhecimento.

Quanto a presença de indicadores de depressão, observou-se em cerca de $44 \%$ dos indivíduos apresentavam indicadores de depressão. O estudo de Pinheiro et al. (2002) também teve observação semelhante, com a presença indicativa de depressão em $46 \%$ dos idosos internados. A associação da presença de indicadores de depressão em idosos e a mortalidade tem sido identificada em estudos nacionais, mas não foi observada nesse estudo. Pode ainda estar relacionada ao número de dados ausentes e a predominância de indivíduos do sexo masculino, visto que alguns estudos têm observado uma maior associação entre óbito e depressão em indivíduos do sexo feminino.

Em pacientes hospitalizados a presença de Delirium é alta, estando presente em $11 \%$ a $33 \%$ dos pacientes na admissão hospitalar e 3\% a 56\% no período internado (RIGNEY, 2006). Nesse estudo, ela foi observada em 19\% dos idosos, corroborando com achados de F. Pereira e Lopes (2018), que encontraram Delirium em 17,9\% dos idosos internados. Observa-se a associação entre o desfecho de óbito e a presença de Delirium, corroborando com estudo de Ely et al. (2004), que observou a associação da presença de Delirium com aumento da mortalidade, além de maior tempo de permanência no hospital. Quanto ao risco de eventos adversos, verificado pelo ISAR, observou-se a associação entre o escore de alto risco e o desfecho de óbito. Essa associação também tem sido encontrada em estudos internacionais, como visto em Salvi et al. (2008), que identificou $71 \%$ dos idosos avaliados como de alto risco.

Estudar o perfil de saúde da população idosa em ambiente hospitalar e analisar sua mortalidade é uma estratégia para o monitoramento da qualidade da assistência hospitalar prestada. No presente estudo, a mortalidade intra-hospitalar ocorreu em 20,7\% $(n=102)$ dos idosos, corroborando com dados de Cordeiro e Martins (2018), que avaliaram a mortalidade hospitalar de idosos internados na região Sudeste do Brasil, encontrando uma taxa de mortalidade de 11\% em hospitais privados, 12\% nos filantrópicos e $14 \%$ nos hospitais públicos. A maior taxa de mortalidade observada no presente estudo pode estar relacionada ao fato da região central do Rio Grande do Sul, área de abrangência do HUSM, apresentar uma rede de atenção primária em formação, ainda com lacunas na assistência prestada, possibilitando que o acesso à saúde ocorra tardiamente e com situações de maior gravidade.

Destaca-se que o encontrado neste estudo vai ao encontro dos achados na literatura, exceto pelo gênero de prevalência de ingresso e a idade, o qual pode ser justificado pela forma de ingresso ser por patologias agudas como em doenças gástricas, fratura de fêmur, ou por neoplasia, onde o HUSM é 
referência de atendimento tanto na área de traumatologia ou na oncologia. Além deste fato, encontramos achados que retratam que os idosos que ingressam na emergência hospitalar apresentam algum grau fragilidade, assim elevando o risco de desfecho adverso durante a internação, enfatizando o papel desta síndrome nos desfechos durante a internação.

O presente estudo apresentou limitações devido à dificuldade na obtenção de alguns dados, justificada por características próprias do ambiente de um pronto socorro, como alta rotatividade dos pacientes, dinâmica de transferências e ausência, por vezes, de responsáveis para a informação dos dados em tempo hábil. Outra limitação deste estudo foi devido ao acompanhamento da evolução clínica do paciente ter sido realizada através da revisão de prontuários, ficando dependente do preenchimento adequado dos mesmos.

\section{Conclusão}

Identificamos que os idosos que ingressaram na emergência possuíam características semelhantes à literatura, com predomínio do sexo masculino e que tinham algum familiar responsável. Entretanto, o fato do perfil de internação ser de idosos jovens tende a confrontar o encontrado na literatura, pois demonstra maior prevalência de neoplasias, fratura de fêmur e doenças gástricas, o que pode interferir neste achado. Além deste fato, o perfil clínico funcional identificado tem a fragilidade sendo um marcador importante dentro da emergência hospitalar, com alta prevalência nos idosos analisados, bem com o risco de desenvolver algum desfecho adverso, demonstrando que o ingresso de idosos na internação são de alta complexidade e com perdas clinico-funcionais importantes, e que podem torna-los ainda mais dependentes dos serviços assistenciais, o que ocasiona maior gasto com a saúde.

Verificou-se que a população idosa que ingressa em emergência hospitalar apresenta, além da fragilidade e dos riscos de evento adverso, multimorbidades, declínios funcionais e maior exposição a eventos adversos, entre eles o óbito. Dessa forma, sugere-se uma melhor abordagem durante o período de internação que vise à predição de riscos de complicações intra-hospitalares e desfechos desfavoráveis como uma estratégia para modificar essa realidade. Uma das possíveis estratégias é a elaboração de ferramentas de fácil aplicação, próprias para emergência hospitalar e para a realidade da saúde pública brasileiro, onde o risco seja mensurado e estabelecido um protocolo individualizado para o tratamento adequado deste idoso. 


\title{
abstract
}

Population aging is permeated by a series of challenges for Brazilian public health. In this way, considering the presence of comorbidities, functional parameters and presence of fragility, the evaluation of the profile of older adults in hospital admission becomes essential as a way of subsidizing elaboration of strategies. So that the care provided in this environment contemplates the peculiarities of this age group enables better health outcomes. In this way, the profile of older people in admission, hospital evolution and outcome is evaluated. The association of the admission profile with the outcome of hospitalization is also checked. Hence, it is a prospective cohort study. The sociodemographic profile and the clinical-functional profile were used as variables of hospital admission, as variables of the evolution of hospital stay and complications and as outcome hospital discharge or death. Descriptive analysis and chi-square test or Fisher's exact test were performed for association of variables. A total of 493 elderly individuals, most of whom were young and living alone, were admitted to the hospital because of neoplasia. There was a positive association of death with age, CAM, ICC, ISAR, EFE, and CP. It was verified that the older adult population that entered the hospital emergency presented multimorbidities, functional declines, fragility and greater exposure to adverse events, among them death.

keywords

Aging. Hospitalization. Mortality.

\author{
referências
}

\begin{abstract}
ALMAGRO, Pere et al. Multimorbidity gender patterns in hospitalized elderly patients. PLOS ONE, California, v. 15, n. 1, 2020. Disponível em: http://dx.doi.org/10.1371/journal. pone.0227252. Acesso em: 12 maio 2019.
\end{abstract}

BAl, Jianzhong et al. Association between postoperative Delirium and mortality in elderly patients undergoing hip fractures surgery: a meta-analysis. Osteoporosis International, New York, v. 31, p. 317-326, 2019. Disponível em: http://dx.doi.org/10.1007/s00198019-05172-7. Acesso em: 12 maio 2019. 
BRASIL. Ministério da Saúde. Conselho Nacional de Saúde. Dados dos serviços de saúde no Brasil. Brasília, DF: Ministério da Saúde, 2017. Disponível em: http://www. cns.org.br/links/DADOS_DO_SETOR.htm. Acesso em: 12 maio 2019.

CARVALHO, Tatiane Cristina et al. Impacto da hospitalização na funcionalidade de idosos: estudo de coorte. Revista Brasileira de Geriatria e Gerontologia, Rio de Janeiro, v. 21, n. 2, p. 134-142, abr. 2018.

CASTELO, Milena Sampaio et al. Validity of the Brazilian version of the Geriatric Depression Scale (GDS) among primary care patients. International Psychogeriatrics, Fortaleza, v. 22, n. 1, p. 109-113, 3 nov. 2009.

CESAR, Juraci et al. Perfil dos idosos residentes em dois municípios pobres das regiões Norte e Nordeste do Brasil: resultados de estudo transversal de base populacional. Cadernos de Saúde Pública, Rio de Janeiro, v. 24, n. 8, p. 1835-1845, ago. 2008.

CHODOS, Anna et al. Hospitalization-associated disability in adults admitted to a safety-net hospital. Journal of General Internal Medicine, New York, v. 30, n. 12, p. 1765-1772, May 2015. Disponível em: http://dx.doi.org/10.1007/s11606-015-3395-2. Acesso em: 12 maio 2019.

$\mathrm{CIRO}$, Carrie et al. Patterns and correlates of depression in hospitalized older adults. Archives of Gerontology and Geriatrics, Amsterdam, v. 54, n. 1, p. 202-205, 2012.

CORDEIRO, Paula; MARTINS, Mônica. Mortalidade hospitalar em pacientes idosos no Sistema Único de Saúde, região Sudeste. Revista de Saúde Pública, São Paulo, v. 52, p. 1-13, jul. 2018. Disponível em: http://dx.doi.org/10.11606/s1518-8787.2018052000146. Acesso em: 12 maio 2019.

COSTA-JÚNIOR, Florêncio Mariano da; MAIA, Ana Cláudia Bortolozzi. Concepções de homens hospitalizados sobre a relação entre gênero e saúde. Psicologia: Teoria e Pesquisa, Brasília, v. 25, n. 1, p. 55-63, mar. 2009. Disponível em: http://dx.doi. org/10.1590/s0102-37722009000100007. Acesso em: 12 maio 2019.

CULLUM, Sarah et al. Does depression predict adverse outcomes for older medical inpatients? A prospective cohort study of individuals screened for a trial. Age and Ageing, London, v. 37, n. 6, p. 690-695, 2008.

DANIELEWICZ, Ana Lúcia; BARBOSA, Aline Rodrigues; DUCA, Giovâni Firpo del. Nutritional status, physical performance and functional capacity in an elderly population in southern Brazil. Revista da Associação Médica Brasileira, São Paulo, v. 60, n. 3, p. 242-248, June 2014. Disponível em: http://dx.doi.org/10.1590/1806-9282.60.03.0013. Acesso em: 12 maio 2019.

ELY, Wesley et al. Delirium as a predictor of mortality in mechanically ventilated patients in the Intensive Care Unit. JAMA, Chicago, v. 291, n. 14, p. 1753-1762, April 2004. Disponível em: http://dx.doi.org/10.1001/jama.291.14.1753. Acesso em: 12 maio 2019.

ENSRUD, Kristine et al. Frailty and risk of falls, fracture, and mortality in older women: the study of osteoporotic fractures. The Journals of Gerontology Series A: Biological Sciences and Medical Sciences, Oxford, v. 62, n. 7, p. 744-751, July 2007. Disponível em: http://dx.doi.org/10.1093/gerona/62.7.744. Acesso em: 12 maio 2019.

FREIRE, Júlio César Guimarães et al. Fatores associados à fragilidade em idosos hospitalizados: uma revisão integrativa. Saúde Debate, Rio de Janeiro, v. 41, n. 115, p. 1199-1211, dez. 2017. Disponível em: https://doi.org/10.1590/0103-1104201711517. Acesso em: 2 mar. 2020.

GASSMANN, Karl; RUPPRECHT, Roland; FREIBERGER, Ellen. Predictors for occasional and recurrent falls in community-dwelling older people. Zeitschrift für Gerontologie + Geriatrie, Switzerland, v. 42, n. 1, p. 3-10, 2009. 
GRONEWOLD, Janine et al. Identification of hospitalized elderly patients at risk for adverse in-hospital outcomes in a university orthopedics and trauma surgery environment. PLOS ONE, California, v. 12, n. 11, 2017. Disponivel em: https://doi.org/10.1371/ journal.pone.0187801. Acesso em: 12 maio 2019

HAMMOND, Angela et al. Factors associated with persistent risk of depression in older people following discharge from an acute cardiac unit. International Psychogeriatrics, Cambridge, v. 20, n. 4, p. 738-751, 2008.

JIN, Zhaosheng et al. Postoperative Delirium: the findings from a multidisciplinary survey. Psychogeriatrics, Hoboken, v. 20, n. 4, p. 4-11, 2020. Disponível em: https:// doi.org/10.1111/psyg.12518. Acesso em: 12 maio 2019

LANDI, Francesco et al. Calf circumference, frailty and physical performance among older adults living in the community. Clinical Nutrition. Amsterdam, v. 33, n. 3, p. 539-544, June 2014. Disponível em: http://dx.doi.org/10.1016/j.clnu.2013.07.013. Acesso em: 12 maio 2019

LOHMAN, Timothy; ROCHE, Alex; MARTORELL, Reynaldo. Anthropometric standardization reference manual. Chicago: Human Kinetics Books, 1988.

MAEDA, Keisuke et al. Predictive accuracy of calf circumference measurements to detect decreased skeletal muscle mass and European Society for Clinical Nutrition and Metabolism-defined malnutrition in hospitalized older patients. Annals of Nutrition and Metabolism, Basel, v. 71, n. 1/2, p. 10-15, 2017. Disponível em: http://dx.doi. org/10.1159/000478707. Acesso em: 12 maio 2019.

MARTINS, Monica. Uso de medidas de comorbidades para predição de risco de óbito em pacientes brasileiros hospitalizados. Revista de Saúde Pública, São Paulo, v. 44, n. 3, p. 448-456, jun. 2010. Disponivel em: http://dx.doi.org/10.1590/s003489102010005000003. Acesso em: 12 maio 2019.

MENDES, Antonio da Cruz et al. Assistência pública de saúde no contexto da transição demográfica brasileira: exigências atuais e futuras. Cadernos de Saúde Pública, Rio de Janeiro, v. 28, n. 5, p. 955-964, 2012

MIYATA, Hiroaki et al. Performance of in-hospital mortality prediction models for acute hospitalization: Hospital Standardized Mortality Ratio in Japan. BMC Health Services Research, New York, v. 8, n. 1, p. 1-9, Nov. 2008. Disponível em: http://dx.doi. org/10.1186/1472-6963-8-229. Acesso em: 12 maio 2019.

MORLEY, John et al. Frailty consensus: a call to action. Journal of the American Medical Directors Association, Amsterdam, v. 14, n. 6, p. 392-397, June 2013. Disponível em: http://dx.doi.org/10.1016/j.jamda.2013.03.022. Acesso em: 12 maio 2019

MOTTA, Luciana Branco da. Levantamento do perfil de idosos internados em um hospital geral: análise do processo de internação frente às demandas da população geriátrica. Textos sobre Envelhecimento, Rio de Janeiro, v. 3, n. 6, p. 47-77, 2001.

PAPALÉO NETTO, Matheus et al. Delirium. In: CARVALHO FILHO, Eurico Thomaz: PAPALÉO NETTO, Matheus. Geriatria: fundamentos, clínica e terapêutica. 2. ed. São Paulo: Editora Atheneu, 2005. p. 171-180.

PEREIRA, Déborah Santana; NOGUEIRA, Júlia Aparecida Devidé; SILVA, Carlos Antonio Bruno da. Quality of life and the health status of elderly persons: a population-based study in the central sertão of Ceará. Revista Brasileira de Geriatria e Gerontologia, Rio de Janeiro, v. 18, n. 4, p. 893-908, Dec. 2015. Disponível em: http://dx.doi. org/10.1590/1809-9823.2015.14123. Acesso em: 12 maio 2019.

PEREIRA, Esdras et al. Funcionalidade global de idosos hospitalizados. Revista Brasileira de Geriatria e Gerontologia, Rio de Janeiro, v. 17, n. 1, p. 165-176, 2014. 
PEREIRA, Fernando de Bortoli; LOPES, Marcos Antonio. Delirium in elderly inpatients admitted to clinical wards: prevalence and investigation of clinical conditions in a Brazilian sample. Dementia \& Neuropsychologia, Rio de Janeiro, v. 12, n. 2, p. 152-156, June 2018. Disponível em: http://dx.doi.org/10.1590/1980-57642018dn12-020007. Acesso em: 12 maio 2019.

PÉREZ-ZEPEDA, Mario; GUTIÉRREZ-ROBLEDO, Luis Miguel. Calf circumference predicts mobility disability: a secondary analysis of the Mexican health and ageing study. European Geriatric Medicine, Amsterdam, v. 7. n. 3, p. 262-266, June 2016. Disponivel em: http://dx.doi.org/10.1016/j.eurger.2016.01.004. Acesso em: 12 maio 2019.

PIERLUISSI, Edgar et al. Depressive symptoms after hospitalization in older adults: function and mortality outcomes. Journal of the American Geriatrics Society, New York, v. 60, n. 12, p. 2254-2262, 2012.

PINHEIRO, Rejane Sobrino et al. Gênero, morbidade, acesso e utilização de serviços de saúde no Brasil. Ciência \& Saúde Coletiva, Rio de Janeiro, v. 7, n. 4, p. 687-707, 2002. Disponível em: http://dx.doi.org/10.1590/s1413-81232002000400007. Acesso em: 12 maio 2019.

REICHARDT, Lucienne et al. Trajectories of cognitive-affective depressive symptoms in acutely hospitalized older adults: the hospital-ADL study. Journal of Psychosomatic Research, Amsterdam, v. 120, p. 66-73, 2019. Disponivel em: http://dx.doi.org/10.1016/j. jpsychores.2019.03.011. Acesso em: 12 maio 2019.

REIS, Luciana Araújo dos et al. Prevalência e padrão de distribuição do acidente vascular encefálico em idosos submetidos a tratamento fisioterapêutico no município de Jequié, BA. Revista Brasileira de Geriatria e Gerontologia, Rio de Janeiro, v. 11, n. 3, p. 369-378, dez. 2008. Disponível em: http://dx.doi.org/10.1590/1809-9823.2008.11036. Acesso em: 12 maio 2019.

RIGNEY, Ted. Delirium in the hospitalized elder and recommendations for practice. Geriatric Nursing, Amsterdam, v. 27, n. 3, p. 151-157, May 2006. Disponível em: http:// dx.doi.org/10.1016/j.gerinurse.2006.03.014. Acesso em: 12 maio 2019.

RITT, Martin et al. Comparing the predictive accuracy of frailty, comorbidity, and disability for mortality: a 1-year follow-up in patients hospitalized in geriatric wards. Clinical Interventions in Aging, Bethesda, v. 12, n. 293-304, p. 293-304, 2017.

ROLFSON, Darryl et al. Validity and reliability of the Edmonton Frail Scale. Age And Ageing, London, v. 35, n. 5, p. 526-529, June 2006. Disponível em: http://dx.doi. org/10.1093/ageing/afl041. Acesso em: 12 maio 2019.

SALES, Fabricia; SANTOS, Iraci. Perfil de idosos hospitalizados e nível de dependência de cuidados de enfermagem: identificação de necessidades. Texto \& Contexto Enfermagem, Florianópolis, v. 16, n. 3, p. 495-502, 2007.

SALVI, Fabio et al. A geriatric emergency service for acutely ill elderly patients: pattern of use and comparison with a conventional emergency department in Italy. Journal of the American Geriatrics Society, New York, v. 56, n. 11, p. 2131-2138, Nov. 2008. Disponível em: http://dx.doi.org/10.1111/j.1532-5415.2008.01991.x. Acesso em: 12 maio 2019.

SANTOS, Camila de Souza dos; BESSA, Thaíssa Araujo de; XAVIER, André Junqueira. Fatores associados à demência em idosos. Ciência \& Saúde Coletiva, v. 25, n. 2 , p. 603-611, 2020. ISSN 1678-4561. Disponível em: https://doi.org/10.1590/141381232020252.02042018. Acesso em: 2 mar. 2020.

SANTOS, Liara et al. Fontes potenciais de agentes causadores de infecção hospitalar: esparadrapos, fitas adesivas e luvas de 52 procedimento. Revista Panamericana de Infectología, São Paulo, v. 12, n. 3, p. 8-12, 2010. 
SILVA, Eliézer et al. Prevalência e desfechos clínicos de infecções em UTIs brasileiras: subanálise do estudo EPIC II. Revista Brasileira de Terapia Intensiva, São Paulo, v. 24, n. 2, p. 143-150, jun. 2012. Disponível em: http://dx.doi.org/10.1590/s0103507×2012000200008. Acesso em: 12 maio 2019.

SOCIEDADE BRASILEIRA DE GERIATRIA E GERONTOLOGIA (SBGG). Envelhecimento no Brasil e saúde do idoso: SBGG divulga carta aberta à população. Rio de Janeiro: SBGG, 2014. Disponível em: https://sbgg.org.br/envelhecimento-no-brasil-e-saudedo-idoso-sbgg-divulga-carta-aberta-a-populacao-2. Acesso em: 12 maio 2019.

SOURDET, Sandrine; LAFONT, Christine; ROLLAND, Yves; NOURHASHEMI, Fati; ANDRIEU, Sandrine; VELLAS, Bruno. Preventable iatrogenic disability in elderly patients during hospitalization. JAMDA, Columbia, MD, v. 16, n. 8, p. 674-681, Aug. 2015. Disponível em: http://dx.doi.org/10.1016/j.jamda.2015.03.011. Acesso em: 12 maio 2019.

SOUSA-MUÑOZ, Rilva Lopes de et al. Impacto de multimorbidade sobre mortalidade em idosos: estudo de coorte pós-hospitalização. Revista Brasileira de Geriatria e Gerontologia, Rio de Janeiro, v. 16, n. 3, p. 579-589, set. 2013. Disponível em: http:// dx.doi.org/10.1590/s1809-98232013000300015. Acesso em: 12 maio 2019.

TANAKA, Lilian Maria Sobreira et al. Delirium in intensive care unit patients under noninvasive ventilation: a multinational survey. Revista Brasileira de Terapia Intensiva, São Paulo, v. 27, n. 4, p. 360-368, 2015. Disponível em: http://dx.doi.org/10.5935/0103507x.20150061. Acesso em: 12 maio 2019.

TAVARES, João; GRÁCIO, Joana; NUNES, Lisa. Predictive validity of the Identification of Seniors at Risk - Hospitalized Patient tool for identifying functional decline. Revista de Enfermagem Referência, Coimbra, v. 123, n. 15, p. 145-154, Dec. 2017. Disponível em: http://dx.doi.org/10.12707/riv17049. Acesso em: 12 maio 2019.

THIEME, Rubia Daniela et al. Elaboração e implantação de protocolo de alta responsável para idosos com doenças crônicas hospitalizados e com necessidades alimentares especiais. Demetra: Alimentação, Nutrição \& Saúde, Rio de Janeiro, v. 9, p. 269-286, jul. 2014. Disponível em: http://dx.doi.org/10.12957/demetra.2014.10347. Acesso em: 12 maio 2019.

ZISBERG, Anna et al. Hospital-associated functional decline: the role of hospitalization processes beyond individual risk factors. Journal of the American Geriatrics Society, New York, v. 63, n. 1, p. 55-62, 2015

Data de Submissão: 29/05/2019

Data de Aprovação: 13/04/2020 